\title{
Análise da variabilidade genética de arnica (Lychnophora ericoides Less. - Asteraceae) usando marcadores RAPDs
}

\author{
Luciana Queiroz Melo ${ }^{1}$, Ana Yamagushi Ciampi² e Roberto Fontes Vieira ${ }^{2,3}$
}

Recebido em 5/12/2007. Aceito em 15/07/2008

\begin{abstract}
RESUMO - (Análise da variabilidade genética de arnica (Lychnophora ericoides Less.- Asteraceae) usando marcadores RAPDs). O objetivo deste trabalho foi analisar e quantificar a variabilidade genética entre e dentro das populações de arnica por meio de marcadores RAPD. Foram amostradas quatro populações na região geoeconômica do Distrito Federal: Parque Nacional de Brasília (2), Fazenda Água Limpa - UnB (1) e Reserva do Instituto Brasileiro de Geografia e Estatística (IBGE) (1). Folhas de 24 indivíduos de cada região foram coletadas, totalizando 96 indivíduos. Num total de 105 iniciadores testados foram selecionados 15, totalizando 60 bandas polimórficas. Marcadores RAPDs selecionados foram analisados com a utilização dos programas NTSYS e Amova. O dendrograma obtido pelo método UPGMA e coeficiente de dissimilaridade Dice evidenciou quatro agrupamentos consistentes, com índice de dissimilaridade variando entre 62 a 71\%. O teste de Mantel aplicado estabeleceu uma correlação cofenética com valores de $\mathrm{r}=0.82$, significando que as distâncias geográficas entre as populações amostradas estão correlacionadas com a distância genética. A análise de AMOVA mostrou uma percentagem variabilidade genética entre populações de 35,7\% e dentro de populações de 64,3\%, evidenciando uma alta variação entre populações, sendo um importante resultado para definição de uma estratégia de conservação da espécie que se encontra em situação vulnerável à extinção.
\end{abstract}

Palavras-chave: cerrado, marcadores moleculares, plantas medicinais

\begin{abstract}
Genetic variability analysis of arnica (Lychnophora ericoides Less. - Asteraceae) using RAPD markers). The main objective of this research was to analyze and quantify the genetic variability within and between populations of arnica using RAPD markers. Four populations from Federal District area, Brazil were sampled: Parque Nacional de Brasília - (2), Fazenda Água Limpa UnB (1), and Reserva do Instituto Brasileiro de Geografia e Estatística (IBGE) (1). Leaves from twenty-four individuals from each population were collected and preserved under refrigeration. Fifteen primers were selected from 105 tested, totalizing 60 polymorphic bands. Scored RAPD markers were analyzed using NTSYS and Amova. The results indicated four consistent clusters, with dissimilarity index varying from 62 to 71\%. The Mantel test indicates a cophenetic correlation (r-0.82), which means that the geographic distances are correlated to the genetic distances. An AMOVA analysis presented 35.7\% variation among populations, and 64.3\% within populations, showing a high variation among populations. This is an important result for conservation strategy for such species considered vulnerable to extinction.
\end{abstract}

Key words: cerrado, medicinal plants, molecular markers

\section{Introdução}

A conservação de plantas medicinais do Cerrado é fundamental como estratégia para garantir a sobrevivência de sua variabilidade genética, possibilitando futuros trabalhos de prospecção gênica e de metabólitos secundários. O cerrado contém uma flora medicinal riquíssima, com cerca de 700 espécies utilizadas na medicina popular (R.F. Vieira, dados não publicados), porém a grande maioria destas espécies requer de estudos básicos que viabilizem sua conservação.

Lychnophora ericoides Martius é uma espécie arbustiva, de até $3,0 \mathrm{~m}$ de altura com fruto do tipo aquênio, possui folhas lineares e estreitas (1-3 mm) e inflorescência formada por glomérulos contendo capítulos de 3-5 flores violáceas (Semir 1993). Ocorre nos estados de Minas Gerais, Goiás e Distrito Federal, em elevações que variam de 950 a 1.800 m (Dias 1992; Almeida et al. 1998). Cresce em depósitos de minérios de ferro-manganês, afloramentos rochosos de arenito e quartzito, precipícios, rampas rochosas e planaltos de campo rupestre, e campos de pastejo e cerrado (Coyle \& Jones 1981). Apresenta distribuição agregada formando populações definidas espacialmente, com adaptações a ambientes com clima sazonal com eventos periódicos de fogo.

\footnotetext{
1 Companhia Nacional de Abastecimento - Conab, Ministério da Agricultura, Pecuária e Abastecimento

2 Embrapa Recursos Genéticos e Biotecnologia, C. Postal 02372, 70770-900 Brasília, DF, Brasil

3 Autor para correspondência: rfvieira@ cenargen.embrapa.br
} 
260 Melo, Ciampi \& Vieira: Análise da variabilidade genética de arnica (Lychnophora ericoides Less.- Asteraceae)...

O sistema de reprodução de Lychnophora ericoides ainda não é completamente conhecido. L. ericoides apresenta flores agrupadas em capítulos de três a cinco flores e estes reunidos em incapitulescências. As flores apresentam protandria, e a floração ocorre entre os meses de julho a início de outubro e de janeiro a meados de março. L. ericoides pode ser caracterizada como espécie perenifólia, com a brotação de folhas mais intensa entre os meses de julho e setembro e a partir de outubro a meados de janeiro (Avelino 2005). O número de cromossomos em Lychnophora ericoides é $2 \mathrm{n}=34$ (Esteves et al. 2007).

Lychnophora ericoides é conhecida como arnica de Goiás, arnica do campo, candeia, candieiro, pau de candeia e veludinho, e é utilizada tradicionalmente na forma de tintura para o tratamento de hematomas, contusões, dores musculares, varizes e também como anti-inflamatório (Almeida et al. 1998; Souza \& Felfili 2006), cujas folhas são largamente comercializadas nos mercados locais (Cerqueira et al. 1987; Borsato et al. 2000; Kanashiro et al. 2004). Diversas formas farmacêuticas a base de arnica são comercializadas, entre estes, o chá, creme, extrato, gel, pomada e tintura-mãe.

Esta espécie apresenta um intenso polimorfismo (Semir 1993), com alguns espécimes não apresentando nenhum aroma, com folhas verde-esbranquiçadas, intensamente pilosas e menores, e com ramos eretotortuosos, delicados e ramificados, algumas vezes conhecidas como "arnica branca". Outro tipo de arnica apresenta aroma característico e agradável, com folhas maiores de coloração verde intensa, menos pilosas, com diferentes escalas de cor, e com ramos eretos e robustos. Na medicina tradicional existe uma preferência pelo tipo aromático, embora a arnica branca também seja utilizada quando da escassez da outra.

Os constituintes característicos de Lychnophora são lactonas sesquiterpênicas (Borella et al. 1998; Sakamoto et al. 2003), além de flavonoides com efeitos antiinflamatorios (Gobbo Neto et al. 2005), triterpenos (Borella et al. 1998; Sargenti \& Vichnewski 2000), lignanas com atividade analgésica (Borsato et al. 2000). Em outras espécies de Lychnophora, como L. salicifolia, detectou-se ação anti-inflamatória (Miguel et al. 1996) e em extratos de L. rupestris, L. staavioides e L. diamantinana foi relatada atividade analgésica e anticonvulsivante (Taleb-Contini et al. 2008). Além disso, descobriu-se que as raízes e as folhas produzem substâncias anti-inflamatória, enquanto os analgésicos estão apenas na raiz e o caule não produz substâncias de interesse farmacológico (Lopes 2001; Santos et al. 2005). Outras substâncias presentes em L. ericoides, como o goiasensolido e a centraterina, são inibidores do mensageiro celular responsável pelo início da inflamação, evitando a formação das proteínas que desencadeiam o processo inflamatório (Lopes 2001).

A forte atividade citotóxica das lactonas sesquiterpênicas são correlacionadas com a defesa de plantas contra herbívoros (Schmidt 1999; Picman 1986; Santos et al. 2004). As áreas de ocorrência do gênero Lychnophora estão expostas à alta irradiação de luz ultra violeta, a uma alternância entre períodos de seca e chuva, e periodicidade de ocorrência de queimadas. A co-evolução destas plantas com tais características peculiares, assim como com herbívoros, certamente levaram ao estabelecimento de mecanismos de proteção contra os efeitos deletérios das condições ambientais estressantes, o que pode estar relacionado à produção de compostos citotóxicos e pigmentos protetores de UV, tais como STL e flavonóides, respectivamente (Picman 1986; Watermann 1994; Schmidt 1999; Chicaro et al. 2004).

A exploração extrativista de arnica em seu habitat natural pode levar a espécie à extinção, pois observouse que retiradas menos severas (até 50\% da folhas) não levam o indivíduo a morte, porém prejudica a reprodução destes indivíduos no período seguinte a poda. Mesmo que a poda realizada não seja tão severa, mas se for repetitiva ao longo do tempo, esta poderá levar o indivíduo a morte por exaurir as reservas de nitrogênio e carbono (Silva 2005; Andrade \& Hay 2007). Em virtude de seu endemismo, da coleta predatória e da degradação do meio ambiente, L. ericoides foi incluída na categoria de espécie "Vulnerável (V)" ao processo de extinção, estando a sua população reduzida e em declínio (Sociedade Botânica do Brasil 1992).

Lychnophora ericoides possui sementes ortodoxas e pode ser conservada a longo prazo em condições $e x$ situ (L.Q. Melo, dados não publicados). Esta informação permite a coleta de sementes de espécies considerada vulnerável para sua conservação a longo prazo. No entanto, é importante que a estratégia de coleta seja definida também por informações genéticas, permitindo uma melhor representatividade das amostras a serem conservadas.

A técnica de RAPD (Random Amplified Polymorphic DNA) é uma técnica relativamente barata, frente à quantidade de informações genéticas obtidas em curto prazo, podendo ser aplicada a qualquer espécie, envolvendo amostragens não-destrutivas e gerando informações interpretáveis geneticamente, atendendo de forma rápida às demandas existentes em programas de conservação de espécies ameaçadas (Ferreira \& Grattapaglia 1996). Esta já foi usada em análise genética de diversas plantas medicinais, tais como equinácea (Echinacea spp.) (Wolf et al. 1999), manjericão, ou 
alfavaca, (Ocimum spp.) (Vieira et al. 2003), e ginseng (Panax quinquelifolius) (Dapeng et al. 1996; Boehm et al. 1999), permitindo uma boa discriminação dos materiais estudados. Além disso, RAPD tem sido utilizado para discriminação de adulterações em drogas vegetais (Wolf et al. 1999), o que poder ser importante no caso da arnica, pois existem diversas espécies de Asteraceae sendo utilizadas popularmente com este nome, e esta técnica permitiria uma identificação das drogas utilizadas nas farmácias e no comércio.

O objetivo deste trabalho é a análise genética de arnica (L. ericoides) usando marcadores RAPD, visando quantificar a variabilidade genética entre e dentro das populações de áreas sob proteção ambiental, que apresentam diferenças morfológicas e de aroma devido à presença de óleos essenciais, gerando subsídios para programas de conservação genética da espécie.

\section{Material e métodos}

Coleta das amostras - Foram coletadas amostras de folhas expandidas adultas e sadias de indivíduos procedentes de quatro populações da região do Distrito Federal (Fig. 1, Tab.1), conforme descrito a seguir:

0 - Fazenda Água Limpa (FAL), Universidade de Brasília: população próxima ao mirante, em área experimental e próxima a rodovia, sujeita a coletas esporádicas, muitos

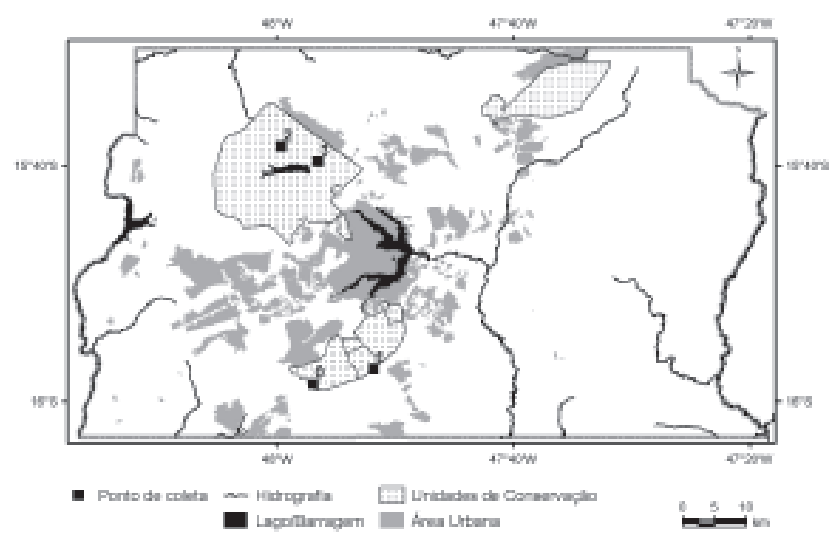

Figura 1. Mapa ilustrando locais de coleta de Lychnophora ericoides Less. na região do Distrito Federal, Brasil. indivíduos (>200), com folhas esverdeadas, aromáticas, compridas, em terreno inclinado.

1 - Instituto Brasileiro de Geografia e Estatística (IBGE): população pequena com poucos indivíduos $(<30)$, agregada, de estatura pequena $(<1,0 \mathrm{~m})$, com aroma não pronunciado, indivíduos em sua maioria em fase vegetativa na coleta de amostras. Folhas pequenas, e esverdeadas; 2 - Parque Nacional de Brasília (APA): população próxima em área fortemente inclinada e pedregosa, com número relativo de indivíduos pequeno (50-70), agregados, de porte maior, observando-se muitos indivíduos adultos, com folhas fortemente aromáticas, pegajosas ao tato. Observou-se também a incidência de insetos predadores dos frutos, o que pode estar causando um declínio nesta população;

3 - Parque Nacional de Brasília (APN): população ocorrendo nas duas margens da estrada, em área inclinada e pedregosa, com número relativo de indivíduos grandes (>200), agregados, com folhas não aromáticas e esbranquiçadas.

Foram consideradas aromáticas as populações cujos indivíduos apresentam óleos voláteis evidentes ao olfato e tato. Amostras de cada população foram coletadas e depositadas no herbário da Embrapa Recursos Genéticos e Biotecnologia (CEN). As distâncias geográficas (em $\mathrm{km}$ ) estabelecidas para entre cada populações foi: APNAPA: 6,1; APN-IBGE: 33,5; APN-FAL:34,8; APA-IBGE :37,5; APA-FAL:37,6; FAL-IBGE:9,5.

Extração e quantificação do DNA - A extração de DNA foi realizada de acordo com o protocolo de Doyle \& Doyle (1987). Após a suspensão do DNA em TE (Tris $\mathrm{HCl} 10 \mathrm{mM}$, EDTA $1 \mathrm{mM}$ ), alíquotas de $2 \mathrm{ml}$ de cada amostra de DNA foram quantificadas por eletroforese em gel de agarose $1,0 \%$ em TBE $1 x$, usando quantidades conhecidas de DNA de fago $\lambda$ : 50, 100, 200 e $400 \mathrm{ng}$, para estimar a quantidade de DNA de cada indivíduo. A corrente usada foi de $72 \mathrm{~V}, 38 \mathrm{~mA}$ por 1 hora. O DNA foi fotografado para a quantificação e diluição $(2,5 \mathrm{ng} / \mathrm{ml})$ de cada amostra.

Seleção de iniciadores e amplificação RAPD - Amplificações foram realizadas em reações com volume total de $13 \mu$ l contendo: $3,42 \mu$ de água MilQ esterilizada;

Tabela 1. Locais de ocorrência de populações de arnica (Lychnophora ericoides Less.) coletada para estudo de diversidade genética.

\begin{tabular}{|c|c|c|c|c|}
\hline \# & Local & N. de indivíduos & Latitude & Longitude \\
\hline 0 & Fazenda Água Limpa, UnB (FAL) & 24 & $15^{\circ} 58^{\prime} 29^{\prime \prime}$ & $47^{\circ} 56^{\prime} 55^{\prime \prime}$ \\
\hline 1 & Instituto Brasileiro de Geografia e Estatística (IBGE) & 24 & $15^{\circ} 54^{\prime} 57^{\prime \prime}$ & $47^{\circ} 53^{\prime} 26^{\prime \prime}$ \\
\hline 2 & Parque Nacional de Brasília (APA) ${ }^{1}$ & 24 & $15^{\circ} 38^{\prime} 18^{\prime \prime}$ & $47^{\circ} 59^{\prime} 36^{\prime \prime}$ \\
\hline 3 & Parque Nacional de Brasília $(\mathrm{APN})^{2}$ & 24 & $15^{\circ} 39^{\prime} 38^{\prime \prime}$ & $47^{\circ} 56^{\prime} 29^{\prime \prime}$ \\
\hline
\end{tabular}

${ }^{1}$ população aromática; ${ }^{2}$ população com indivíduos com características não aromáticas 
262 Melo, Ciampi \& Vieira: Análise da variabilidade genética de arnica (Lychnophora ericoides Less.- Asteraceae)...

$1,04 \mu \mathrm{l}$ de $\operatorname{dNTP}(2,5 \mathrm{mM}) ; 1,04 \mu \mathrm{l}$ de BSA $(2,5 \mathrm{mg} / \mathrm{ml})$, $0,2 \mu \mathrm{l}$ de Taq polimerase ( 5 unidadesg/ $\mu \mathrm{l}$ ), e $3 \mu \mathrm{l}$ de iniciador $(10 \mathrm{ng} / \mu \mathrm{l})$. A quantidade de DNA utilizada foi de $3 \mu \mathrm{l}$ em cada reação na concentração de $2,5 \mathrm{ng} / \mu \mathrm{l}$. Foram preparados mix para 96 reações. As amplificações foram realizadas em termociclador (PTC -100, MJ Research, Inc) com 40 ciclos de desnaturação por 60 minutos a $92{ }^{\circ} \mathrm{C}$, anelamento por 60 minutos a $35^{\circ} \mathrm{C}$, e por final uma extensão por $5^{\prime}$ a $72^{\circ} \mathrm{C}$.

A seleção de iniciadores foi realizado usando duas amostras de cada população testando 105 iniciadores da Operon Tecnology escolhidos ao acaso. Foram selecionados aqueles que apresentaram bandas polimórficas e de melhor intensidade e nitidez quando visualizados em géis de agarose $(1,5 \%)$ com detecção com brometo de etídio e luz ultra violeta, após a eletroforese a $120 \mathrm{~V}$, por 4-5 horas.

Análise dos dados - Em cada reação foram identificadas as bandas polimórficas nos géis de agarose e anotadas como presença (1) ou ausência (0) do fragmento para cada indivíduo, gerando uma matriz de dados com valores binários, 0 e 1 . O coeficiente de Dice (1-DICE) foi utilizado para estimar dissimilaridade genética entre as populações de arnica, sendo obtido o dendrograma pelo método da média aritmética não ponderada para agrupamento aos pares (UPGMA-Unweighted Pair Group Arithmetic Average). A estabilidade dos agrupamentos foi testada por reamostragem pelo procedimento de 10000 bootstrap, utilizando o programa BoodP (Coelho 2002). Foi utilizado o programa NTSYSpc versão 2.1 (Adams et al. 2002). A Análise de Variância Molecular (AMOVA) foi realizada utilizando o programa Arlequin versão 2000 (Schneider et al. 2000).

\section{Resultados e discussão}

Dos 105 iniciadores testados foram selecionados 15 , os quais geraram 60 bandas polimórficas para análise da variabilidade genética de L. ericoides. Foi obtida uma média de 4 bandas/iniciador (Tab. 2) sendo que o tamanho das bandas selecionadas variou entre 450 e 2.036 pares de bases nos diferentes iniciadores (Fig. 2).

A tecnologia RAPD aplicada a outras espécies medicinais e aromáticas como Ginseng (Panax spp.) e Echinacea (Echinacea spp.), evidenciou uma discriminação dos acessos com um número reduzido de iniciadores, com amplificação significativa de fragmentos polimórficos (Wolf et al. 1999).

A Fig. 3 ilustra o agrupamento das populações com alto grau de consistência dos agrupamentos, com evidente separação das populações. Os índices de dissimilaridade de Dice apresentaram variação entre $62 \%$ a $71 \%$, o que demonstra uma dissimilaridade não tão
Tabela 2. Iniciadores utilizados nas reações RAPDs com respectivas sequências de bases e número de bandas polimorficas.

\begin{tabular}{llc}
\hline Iniciador & $\begin{array}{c}\text { Seqüência de bases } \\
\left(5^{\prime} \rightarrow 3^{\prime}\right)\end{array}$ & $\begin{array}{c}\text { N. de bandas } \\
\text { polimórficas }\end{array}$ \\
\hline OPB-10 & CTGCTGGGAC & 3 \\
OPL-05 & ACGCAGGCAC & 3 \\
OPL-19 & GAGTGGTGAC & 4 \\
OPN-02 & ACCAGGGGCA & 6 \\
OPN-06 & GAGACGCACA & 2 \\
OPN-08 & ACCTCAGCTC & 3 \\
OPN-12 & CACAGACACC & 4 \\
OPN-18 & GGTGAGGTCA & 4 \\
OPN-19 & GTCCGTACTG & 3 \\
OPN-20 & GGTGCTCCGT & 3 \\
OPO-19 & GGTGCACGTT & 5 \\
OPV-15 & CAGTGCCGGT & 5 \\
OPV-20 & CAGCATGGTC & 5 \\
OPX-04 & CCGCTACCGA & 5 \\
OPX-06 & ACGCCAGAGG & 5 \\
Total & & 60 \\
\hline
\end{tabular}

elevada $(<75 \%)$ entre populações, embora os dados tenham sido capazes de distinguir as populações estudadas. As populações do Parque Nacional de Brasília (APA) e da Fazenda Água Limpa (FAL) (distantes $37,6 \mathrm{~km}$ ), ambas consideradas aromáticas, apresentaram a menor dissimilaridade de Dice $(62 \%)$, com a consistência do agrupamento de $72,5 \%$. Esta informação coincide com a presença de aroma em folhas de ambas as populações, evidenciando uma maior proximidade genética entre populações com esta característica. A população do Parque Nacional de Brasília onde predominava indivíduos sem aroma (APN), mostrou-se geneticamente um pouco mais dissimilar quando comparada às duas anteriores $(66,5 \%)$, com a consistência do agrupamento de $67,5 \%$. Isto evidencia que embora haja uma maior proximidade geográfica entre as populações do Parque Nacional de Brasília (distantes $6,1 \mathrm{~km}$ ), estas populações não foram agrupadas quanto à dissimilaridade, muito embora a diferença entre valores de dissimilaridade não sejam tão expressivos.

Do ponto de vista químico, Lyra et al. (dados não publicados) observaram a presença de óleos essenciais na população APA, denominada aromática, enquanto a segunda população (APN), praticamente não apresentou óleo essencial. Esse mecanismo de produção de metabólitos secundários pode estar associado à dissimilaridade apresentada (66,5\%), muito embora seja necessário realizar uma avaliação mais detalhada para confirmação. Este fato tem importância quando se pretende amostrar sementes de populações para conservação, devendo considerar uma amostragem para cada população separadamente. Outra questão a ser considerada é o fato de estarem em áreas de preservação 

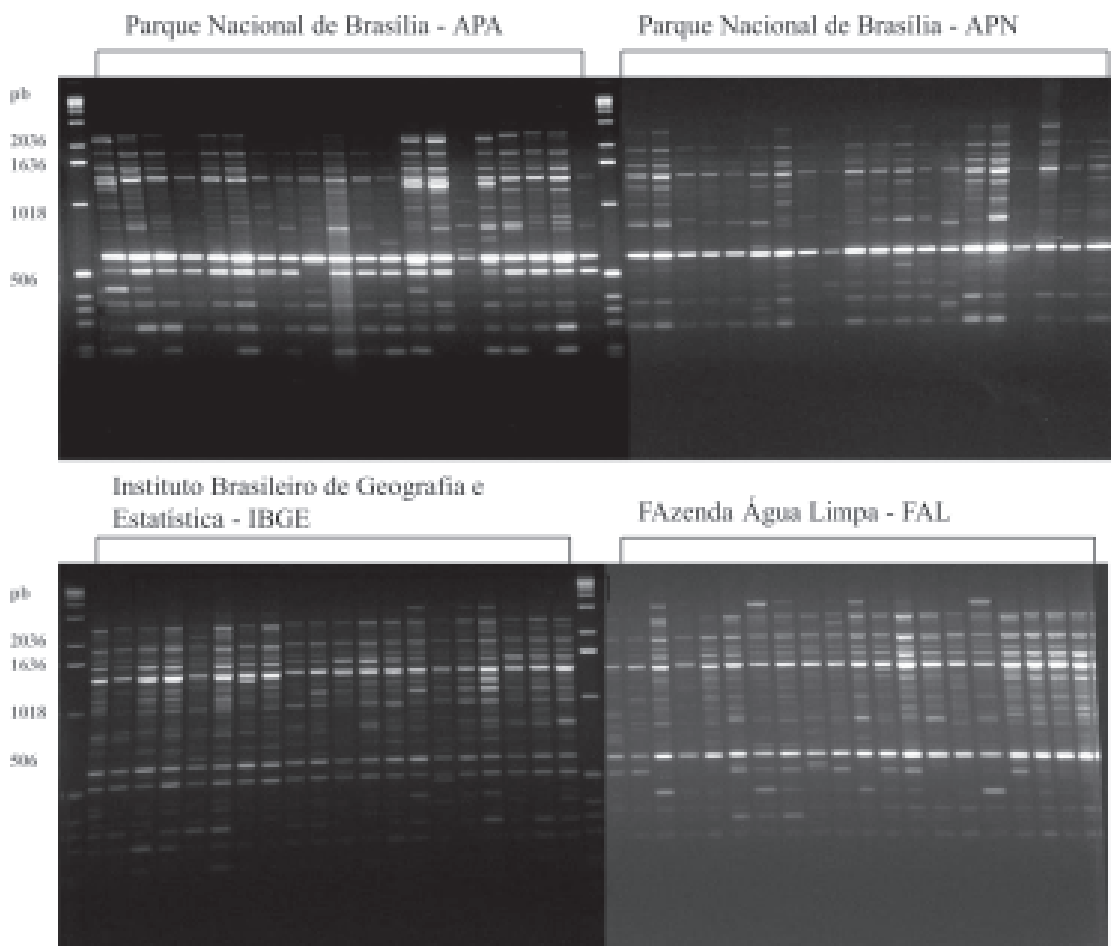

Figura 2. Gel ilustrando RAPD de quatro populações de arnica (Lychnophora ericoides Less.) usando o primer OPX 04. As setas indicam bandas polimórficas.

permanente, eventualmente sujeitas ao fogo e pressão herbívora, o que podem causar uma alteração na produção de metabólitos secundários. Foram observados danos causados por insetos foram observados nas populações do Parque Nacional de Brasília, sendo identificados dois tipos de insetos nas inflorescências: Tomoplagia rupestirs (Diptera: Tephritidae); Malanagromyza minimoides (Diptera: Agromizidae); que danificam a base da inflorescência prejudicando a formação de frutos saudáveis, com baixo rendimento de sementes viáveis e provavelmente prejudicando a

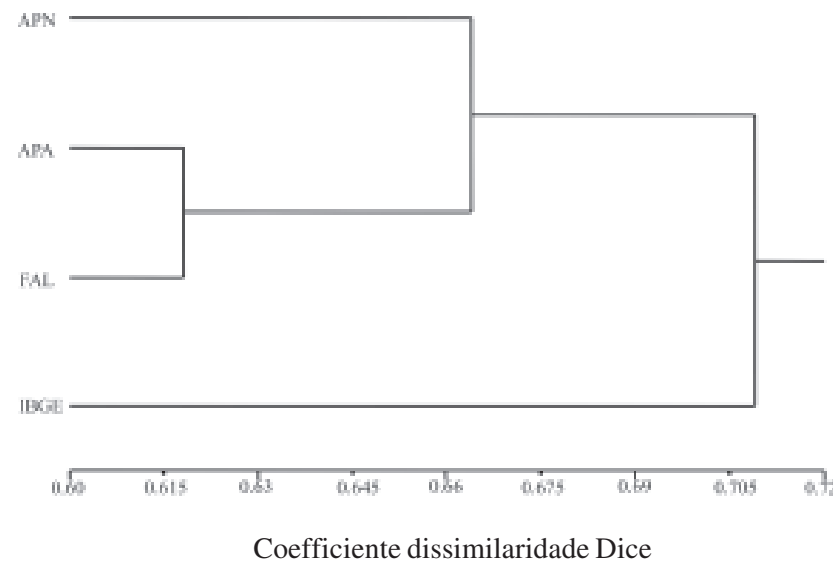

Figura 3. Dendrograma gerado pelo critério de agrupamento UPGMA representando a dissimilaridade de Dice entre quatro populações de arnica (APN e IBGE não aromáticas; FAL e APA aromáticas) analisadas com 60 locos RAPD. formação de novos indivíduos (Vieira et al., dados não publicados).

Recentes estudos indicam um alto grau de variabilidade na quantidade e composição de óleos essenciais nas folhas de populações de Lychnophora oriundas de diferentes locais. Observou-se populações com aroma presente e contendo majoritariamente $\alpha$-bisabolol $(48,2 \pm 15,6 \%)$; um grupo com indivíduos com aroma, porém com alto teor de $o$-cymene $(15,45 \pm 5,8 \%)$ e $\alpha$-thujene $(12.9 \pm 5,5 \%)$, e um terceiro grupo com indivíduos sem aroma (Curado et al. 2006; C.G.V. Lyra et al., dados não publicados).

A população do IBGE formou um agrupamento isolado dos demais, apresentando a maior dissimilaridade em relação às demais populações $(71 \%)$, com consistência de $100 \%$ do agrupamento, estimado pela reamostragem de 10.000 bootstrap. Esta separação era esperada, uma vez que esta população apresenta um hábito distinto das demais (indivíduos $<1,0 \mathrm{~m}$ ), mesmo estando relativamente próxima a população FAL $(9,5 \mathrm{~km})$, a dissimilaridade de Dice agrupou separadamente ambas as populações.

O teste de Mantel aplicado estabeleceu uma correlação cofenética com valores de $r=0.82$. Isto significa que as distâncias entre as populações amostradas estão correlacionadas com a distância genética, indicando que deve ser considerado no processo de coleta de sementes para conservação $e x$ situ um maior número de áreas para representar a maior 
variabilidade possível. Considerando que as sementes de L. ericoides são ortodoxas (Melo 2006), estes resultados possibilitam iniciar um planejamento de coleta das principais populações ainda remanescentes, considerando o alto grau de exploração extrativista encontrado atualmente.

A análise de AMOVA realizada evidenciou uma percentagem de variação de $35,7 \%$ entre populações e de $64,3 \%$ dentro populações, o que demonstra uma alta divergência entre e dentro de populações, confirmando o dendrograma gerado na análise de dissimilaridade (Fig. 3). Segundo Avelino (2005), Lychnophora ericoides mostra-se xenógama facultativa, formando frutos com semente por polinização cruzada, por autopolinização e por agamospermia. Este fato explica a alta variabilidade existente dentro da população. Avelino (2005) registrou visitas de 32 espécies de insetos, além de quatro espécies de beija-flores, e considera as abelhas do gênero Bombus e duas espécies da família Scoliidae como os polinizadores efetivos. A polinização por insetos que não se deslocam por longas distâncias, associada ao fato de que Lychnophora é um gênero de arbustos adaptado a ambientes sazonalmente secos sujeitos às queimadas (o que tornaria as espécies edaficamente restritas a substratos particulares ou a regimes diferenciados de umidade) pode explicar a distribuição restrita da espécie (Almeida et al. 1998), assim como a alta divergência entre populações, que pode ser ocasionada devido ao isolamento existente.

$\mathrm{Na}$ análise de AMOVA entre locais de coleta dois a dois (Tab. 3), verificou-se que as populações APN e IBGE apresentaram os maiores valores quanto à percentagem de variação genética (48\%). A população IBGE foi a que se mostrou mais distante das demais, com 35,9\% e 39\% de variação genética, em relação às populações do APA e FAL, respectivamente. Este resultado confirma a dissimilaridade genética apresentada no dendograma, e era esperado, uma vez

Tabela 3. Distribuição de variabilidade entre e dentro de populações, em quatro populações de Lychnophora ericoides Less., obtida por análise da variância molecular com os dados de RAPD.

\begin{tabular}{lcc}
\hline Fonte de variação & $\begin{array}{c}\% \text { de } \\
\text { variabilidade }\end{array}$ & $\mathrm{P}$ \\
\hline Dentro de cada População & 64,3 & $0,00000 \pm 0,00000$ \\
Entre 4 Populações & 35,7 & $0,00000 \pm 0,00000$ \\
Entre Pop APN $\times$ APA & 25,2 & $0,00000 \pm 0,00000$ \\
Entre Pop APN $\times$ IBGE & 48,0 & $0,00000 \pm 0,00000$ \\
Entre Pop APN $\times$ FAL & 34,9 & $0,00000 \pm 0,00000$ \\
Entre Pop APA $\times$ IBGE & 35,9 & $0,00000 \pm 0,00000$ \\
Entre Pop APA $\times$ FAL & 17,1 & $0,00000 \pm 0,00000$ \\
Entre Pop FAL $\times$ IBGE & 39,0 & $0,00000 \pm 0,00000$
\end{tabular}

que esta população apresenta características de habito observadas bem distintas das demais populações. A menor variação genética entre populações foi apresentada entre as populações APA e FAL, com $17,1 \%$, seguido da variabilidade encontrada entre as populações do Parque Nacional (APA e APN), com 25,2\% Embora as populações APA e FAL apresentem como característica similar a presença de aroma nas folhas, e isso se reflita no agrupamento baseado em dissimilaridade (Fig. 3), as variações genéticas apresentadas corroboram a necessidade de se amostrar para conservação ex situ, cada população individualmente. Tem sido frequentemente reportado na literatura a avaliação de populações quimicamente distintas, em espécies como Tomilho (Thymus vulgaris L.) (Echeverrigaray et al. 2001), Hesperozygis ringens Benth. (Fracaro \& Echeverrigaray 2006), Piper aduncum L. (Potzernheim et al. 2006), entre outras, formando quimiotipos, e onde a avaliação através de marcadores RAPDs tem confirmado esta separação, embora estes marcadores não estejam necessariamente associados a este perfil químico (Vieira et al. 2003a; b; Vieira \& Simon 2006).

A hipótese proposta é que estas populações de arnica, com predominância de fecundação cruzada, devem ter uma alta heterozigose dos genes que controlam a biossíntese destes constituintes, o que se torna evidente quando encontramos indivíduos aromáticos, e dentre estes, quimiotipos diversos, ou mesmo indivíduos sem aroma. Este fato contrasta com espécies aromáticas e medicinais, selecionadas e domesticadas, como por exemplo, espécies de Mentha, Ocimum, entre outras, que apresentam quimiotipos definidos e homogêneos, fruto de seleção pelo homem através de muitos séculos.

Os resultados obtidos com a técnica RAPD demonstram a clara separação genética entre as populações de arnica. A alta da variação genética entre populações tem implicação direta para a proposta de conservação, indicando uma coleta de sementes de grande número de populações, no sentido de salvaguardar maior diversidade genética existente da espécie. Além disso, o estudo com marcadores moleculares podem no futuro gerar marcadores que possibilitem a autenticar e rastrear a origem da matéria prima para fitomedicamentos derivados desta espécie (Percifield et al. 2007). O impacto do extrativismo requer também orientação técnica aos coletores de arnica, de maneira a possibilitar um manejo da espécie dentro das normas adequadas (Andrade \& Hay 2007). Os modelos de preservação e manejo sustentado de $L$. ericoides devem considerar todos os aspectos de variação da espécie, existentes nos diferentes locais de ocorrência. 


\section{Agradecimentos}

Este trabalho foi financiado com recursos do Fundo Nacional do Meio Ambiente (FNMA) e EMBRAPA. Os autores agradecem aos gerentes do Parque Nacional de Brasília, IBAMA/DF, do Instituto Brasileiro de Geografia e Estatística (IBGE) e da Fazenda Água Limpa, UnB, Brasília, DF, pela permissão na coleta de amostras para estudo; à Zilneide P.S. Amaral e Aécio A. dos Santos, pelo auxílio durante as atividades de laboratório e coleta de material em campo.

\section{Referências bibliográficas}

Adams, D.; Kim, J.; Jensen, R.; Marcus, L.; Slice, D.E. \& Walker, J. 2002. NTSYSpc: Numerical Taxonomy and Multivariate Analysis System, versão 2.10z @ by Applied Biostatistics, Inc.

Almeida, S.P.; Proença, C.E.B.; Sano, S.M. \& Ribeiro, J.F. 1998. Cerrado: espécies vegetais úteis. Planaltina, Embrapa/ CPAC.

Andrade, B.S. \& Hay, J.D. 2007. Estimation of aerial biomass of Lychnophora ericoides (Mart.). Brazilian Archives of Biology and Technology 50: 687-694.

Avelino, A.S. 2005. Biologia reprodutiva de Lychnophora ericoides Mart. (Asteraceae: Vernoniae). Tese de Mestrado. Brasília, Universidade de Brasília.

Boehm, C.L.; Harrison, H.C.; Jung, G. \& Nienhuis, J. 1999. Organization of American and Asian ginseng germplasm using Randomly Amplified polymorphic DNA (RAPD) markers. Journal of the American Society for Horticultural Science 124: 252-256.

Borella, J.C.; Lopes, J.L.C.; Vichnewski, W.; Cunha, W.R. \& Herz, W. 1998. Sesquiterpene lactones, triterpenes and flavones from Lychnophora ericoides and Lychnophora pseudovillosissima. Biochemical Systematics and Ecology 26: 671-676.

Borsato, M.L.C.; Grael, C.F.F.; Souza, G.E.P. \& Lopes, N.P. 2000. Analgesic activity of the lignans from Lychnophora ericoides. Phytochemistry 55: 809-813.

Cerqueira, M.B.S.; Souza, J.T.; Júnior, R.A. \& Peixoto, A.B.F. 1987. Ação analgésica do extrato bruto aquoso liofilizado do caule e folhas da Lychnophora ericoides Mart. Ciência e Cultura 39: 551-553.

Chicaro, P.; Pinto, E.; Colepicolo, P.; Lopes, J.L.C. \& Lopes, N.P. 2004. Flavonoids from Lychnophora passerina (Asteraceae): potential antioxidants and UV-protectants. Biochemical Systematics and Ecology 32: 239.

Coelho, A.S. 2002. BoodP Avaliação dos erros associados a estimativas de distância/similaridades através do procedimento de bootstrap com número de variáveis de marcadores (software). Goiânia, UFG. Instituto de Ciências Biológicas. Laboratório de Genética vegetal.

Coyle, N.C. \& Jones, S.B. 1981. Lychnophora (Compositae; Vernoniae), a genus endemic to the Brazilian planalto. Brittonia 33: 528-542.

Curado, M.A.; Oliveira, C.B.A.; Jesus, J.G.; Santos, S.C.; Seraphin, J.C. \& Ferri, P.H. 2006. Environmental factors influence on chemical polymorphism of the essential oils of Lychnophora ericoides. Phytochemistry 67: 2363-2369.
Dapeng, B.; Brandle, J. \& Reeleder, R. 1996. Genetic diversity in North American ginseng (Panax quinquelifolius L.) grown in Ontario detected by RAPD analisys. Genome 40: 111-115.

Dias, B.F.S. 1992. Cerrado: uma caracterização. Pp. 11-34. In: Instituto Brasileiro do Meio Ambiente e Recursos Naturais / Fundação Pró-Natura. Alternativas de desenvolvimento das cerrados: manejo e conservação dos recursos renováveis. Brasília, DF.

Doyle, J.J. \& Doyle, J.L. 1987. Isolation of plant DNA from fresh tissue. Focus 12: 13-15.

Esteves, M.M.; Martins, E.R.F. \& Semir, J. 2007. Cytotaxonomy of Lychnophora Mart. (Asteraceae, Vernonieae, Lychnophorinae) species. Caryologia 60: 21-28.

Echeverrigaray, S.; Agostini, G.; Atti-Serfini, L.; Paroul, N.; Pauletti, G.F. \& Santos, A.C.A. 2001. Correlation between the chemical and genetic relationships among commercial thyme cultivars. Journal of Agricultural and Food Chemistry 49: 4220-4223.

Ferreira, M.E. \& Grattapaglia, D. 1998. Introdução ao uso de marcadores moleculares em análise genética. $3^{\text {a }}$ ed. Brasília, Embrapa Cenargen.

Fracaro, F. \& Echeverrigaray, S. 2006. Genetic Variability in Hesperozygis ringens Benth. (Lamiaceae), an Endangered Aromatic and Medicinal Plant of Southern Brazil. Biochemical Genetics 44: 479-490.

Gobbo Neto, L.; Santos, M.D.; Kanashiro, A.; Almeida, M.C.; Lucisano-Valim, Y.M.; Lopes, J.L.C.; Souza, G.E.P. \& Lopes, N.P. 2005. Evaluation of the anti-inflammatory and antioxidant activities of di-C-glucosylflavones from Lychnophora ericoides (Asteraceae). Planta Medica 71: 3-6.

Kanashiro, A.; Kabeya, L.M.; Polizello, A.C.M.; Lopes, N.P.; Lopes, J.L.C. \& Lucisano-Valim, Y.M. 2004. Inhibitory activity of flavonoids from Lychnophora sp. on generation of reactive oxygen species by neutrophils upon stimulation by immune complexes. Phytotherapy Research 18: 61-65.

Lopes, N.P. 2001. A essência da arnica. Pesquisa Fapesp 64: 42-44.

Melo, L.Q. 2006. Estratégias para Conservação ex situ de Arnica (Lychnophora ericoides Less) - Asteraceae. Dissertação de Mestrado. Brasília, Universidade de Brasília.

Miguel, O.G.; Lima, E.O.; Morais, V.M.F.; Gomes, S.T.A.; Monache, F.D.; Cruz, A.B.; Cruz, R.C.B. \& Cechinel Filho, V. 1996. Antimicrobial activity of constituents isolated from Lychnophora salicifolia (Asteraceae). Phytotherapy Research 10: 694-696.

Percifield, R.J.; Hawkins, J.S.; Mccoys, J.A.; Widrlechnerz, M.P. \& Wendel, J.F. 2007. Genetic diversity in Hypericum and AFLP markers for species-specific identification of H. perforatum L. Planta Medica 73: 1614-1621.

Picman, A.K. 1986. Biological activities of sesquiterpene lactones. Biochemical Systematics and Ecology 14: 255.

Potzernheim, M.; Bizzo, H.; Costa, T.A.; Vieira, R.F.; Silva, M.C. \& Gracindo, L.A. 2006. Chemical characterization of seven Piper species (Piperaceae) from Federal District, Brazil, based on volatile oil constituents. Revista Brasileira de Plantas Medicinais 8: 10-12.

Sakamoto, H.T.; Flausino, D.; Castellano, E.E.; Stark, C.B.W.; Gates, P.J. \& Lopes, N.P. 2003. Sesquiterpene lactones from Lychonophora ericoides. Journal of Natural Products 66 : 693-695. 
266 Melo, Ciampi \& Vieira: Análise da variabilidade genética de arnica (Lychnophora ericoides Less.- Asteraceae)...

Santos, M.D.; Gobbo Neto, L.; Albarella, L.; Souza, G.E.P. \& Lopes, N.P. 2005. Analgesic activity of di-caffeoylquinic acids from roots of Lychnophora ericoides (Arnica da serra). Journal of Ethnopharmacology 96: 545-549.

Sargenti, S.R. \& Vichnewski, W. 2000. Sonication and liquid chromatography as a rapid technique for extraction and fractionation of plant material. Phytochem Anal 11: 69-73.

Schmidt, TJ. 1999. Toxic activities of sesquiterpene lactones: structural and biochemical aspects. Current Organic Chemistry 3: 577.

Schneider, S.; Roessli, D.E \& Excoffier, L. 2000. Arlequin a software for population genetics data analysis. versão 2.000.

Semir, J. 1993. Revisão taxonômica de Lychnophora Mart. (Vernonieae, Compositae). Tese de Doutorado. Campinas, Universidade de Campinas.

Silva, D.M.S. 2005. Estrutura populacional, fenologia, crescimento e efeito de poda em Lychnophora ericoides Mart. (Compositae: Asteraceae). Tese de Doutorado. Brasília, Universidade de Brasília.

Sociedade Botânica do Brasil. 1992. Centuria Plantarum Brasiliensium Exstintionis Minimata. Brasília, DF, Sociedade Botânica do Brasil.

Souza, C.D. \& Felfili, J.M. 2006. Uso de plantas medicinais na região de Alto Paraíso de Goiás, GO, Brasil. Acta Botanica Brasilica 20: 135-142.
Taleb-Contini, S.H.; Santos, W.F.; Mortari, M.R.; Lopes, N.P. \& Lopes, J.L.C. 2008. Neuropharmacological effects in mice of Lychnophora species (Vernonieae, Asteraceae) and anticonvulsant activity of 4,5-di-O-[E]-caffeoylquinic acid isolated from the stem of L. rupestris and L. staavioides. Basic and Clinical Pharmacology and Toxicology 102: 281-286.

Vieira, R.F.; Goldsbrough, P. \& Simon, J.E. 2003a. Genetic Diversity of Basil (Ocimum spp.) Based on RAPD Markers. Journal of the American Society for Horticultural Science 128: 94-99.

Vieira, R.F.; Paton, A. \& Grayer, R. 2003b. Chemical profiling of Ocimum americanum. Phytochemistry 63: 555-567.

Vieira, R.F. \& Simon, J.E. 2006. Chemical characterization of basil (Ocimum spp.) based on volatile oils. Flavour and Fragance 21: 214-221.

Waterman, P.G. \& Mole, S. 1994. Why are phenolic compounds so important? In Analysis of phenolic plant metabolites. P.G. Waterman \& S. Mole (eds.). Oxford, Blackwell Scientific Publications.

Wolf, H.; Zundorf, I.; Winckler, T.; Bauer, R. \& Dingermann, T. 1999. Characterization o Echinaceae species and detection of possible adulterations by RAPD analisys. Planta Medica 65: 773-774. 